\title{
Cefaleia do tipo tensional: revisão de literatura
}

\author{
Tension-type headache: literature review \\ Cefalea tensional: revisión de la literatura \\ Marina Coimbra da CRUZ \\ Lucas Coimbra da $\mathbf{C R U Z}^{2}$ \\ Marlene Cabral Coimbra da $\mathbf{C R U Z}^{3}$ \\ Renan Paes de CAMARGO ${ }^{1}$ \\ ${ }^{l}$ Graduando (a) do Curso de Medicina da Universidade Camilo Castelo Branco - UNICASTELO \\ Campus Fernandópolis - 15600-000 Fernandópolis-SP, Brasil \\ ${ }^{2}$ Médico Residente em Radiologia do Hospital de Ensino Santa Casa de Misericórdia de Fernandópolis \\ $e$ Centro de Diagnóstico por Imagem de Fernandópolis (CDI) -15600-000 - Fernandópolis-SP, Brasil \\ ${ }^{3}$ Docente dos Cursos de Medicina e Odontologia da Universidade Camilo Castelo Branco - UNICASTELO \\ Campus Fernandópolis - 15600-000 Fernandópolis-SP, Brasil
}

\begin{abstract}
Resumo
Introdução: Cefaleia é uma dor de acometimento cefálico, sendo o sintoma neurológico mais comum e queixa médica muito frequente. Pode ser primária, quando é a patologia em si, ou secundária, quando representa um sintoma de outra patologia estrutural. Objetivo: Apresentar uma síntese das informações mais relevantes sobre a cefaleia tensional. Material e Método: Foi realizada uma revisão em livros e bases de dados eletrônicos, tendo como critério de inclusão artigos tidos como de grande relevância, publicados em português, inglês ou espanhol. Resultados: Destaca-se entre as primárias, a cefaleia tipo tensional, caracterizada por sensações dolorosas de caráter compressivo, leves ou moderadas, nas regiões frontal, parietal, temporal e/ou occipital, bilateralmente. É subclassificada de acordo com sua duração em episódica pouco frequente, episódica frequente e crônica. Sua fisiopatologia é complexa e envolve mecanismos periféricos e centrais de gênese da dor, devendo ser diferenciada de outros tipos de cefaleia. Exibe diversos fatores desencadeantes, em especial os de ordem psicológica, que na maioria dos casos podem ser evitados como forma de prevenção. Sua terapêutica pode ser farmacológica ou não, agindo de forma abortiva e/ou profilática. Conclusão: A cefaleia tensional, embora muito prevalente, é subdiagnosticada, geralmente pela falta de conhecimento de médicos generalistas, fato que pode resultar em adoção de planos terapêuticos indevidos e, consequentemente, culminar em prejuízo na qualidade de vida dos doentes. Este distúrbio é passível de prevenção, com mudanças de hábitos a fím de se minimizar ou extinguir fatores desencadeantes.
\end{abstract}

Descritores: Cefaleia; Cefaleia do Tipo Tensional; Manifestações Neurológicas.

\begin{abstract}
Introduction: Cephalea is a type of cephalic involvement pain, having neurological symptom as the most common aspect and it is a very frequent medical complaint. It can be either primary, when it is itself the pathology, or secondary, when it represents a symptom of another structural pathology. Objective: Showing most relevant information synthesis on tensional cephalea. Material and Method: A review on books and electronic data bases was held, having as inclusion criteria: great relevance articles which were published in Portuguese, English and Spanish. Results: Out of primary cephalea, the tensional one stands out, which is characterized by painful sensations of compressive nature, either light or moderate, on frontal, parietal, temporal and/or occipital, bilaterally. It is sub-classified according to its duration in infrequent, frequent and chronic types. Its therapeutics is complex and involves peripheral and central mechanisms of pain genesis, and it must be distinguished from other types of cephalea. It shows several triggering factors, specially psychological ones, which in most of cases can be prevented as a prophilaxis. Its therapeutics can be pharmacological or not, acting on an abortive and/or prophylactic way. Conclusion: Tensional Cephalea, although very prevalent, is underdiagnosed, generally by lack of knowledge of the general practioneer; this factor can result in adoption of incorrect therapeutical plans and, consequently, lower life quality of ailing patients. Such disorder is likely to be prevented at changing habits in order to minimize or extinguish triggering factors.

Descriptors: Headache; Tension-Type Headache; Neurologic Manifestations.
\end{abstract}

\section{Resumen}

Introducción: Cefalea es una afección de dolor de cabeza, el síntoma neurológico más común y frecuente queja médica. Puede ser primaria, cuando es la condición en sí, o secundaria, cuando se trata de un síntoma de otra patología estructural. Objetivo: Presentar una visión general de las informaciones más relevantes sobre la cefalea tipo tensional. Material y Métodos: Una revisión se llevó a cabo en los libros y bases de datos electrónicas, y que tiene como criterios de inclusión artículos considerados como altamente relevantes, publicados en Portugués, Inglés o Español. Resultados: Se destaca entre las primarias, la cefalea de tipo tensional, que se caracteriza por sensaciones dolorosas de naturaleza compresiva, de intensidad de leve a moderada, que afecta los lóbulos frontal, parietal, temporal y/o occipital, bilateralmente. Además, se clasifican en función de su duración en infrecuente episódica, episódica frecuente y crónica. Su fisiopatología es compleja e implica mecanismos centrales y periféricos de la génesis del dolor, y debe distinguirse de otros tipos de dolor de cabeza. Muestra varios factores desencadenantes, especialmente psicológicos, que en la mayoría de los casos se pueden evitar, como medio de prevención. Su terapia puede ser farmacológica o no, actuando abortiva y/o profilácticamente. Conclusión: cefalea tensional, aunque muy prevalente, está infra diagnosticada, a menudo por falta de conocimiento de los médicos generales, que pueden dar lugar a la consiguiente adopción de planes de tratamiento impropio y por consiguiente culminar en la pérdida de la calidad de vida de los pacientes.

Descriptores: Cefalea; Cefalea de Tipo Tensional; Manifestaciones Neurológicas.

\section{INTRODUÇÃO}

Dor trata-se de um sintoma subjetivo, resultante de uma combinação de fatores biológicos, psicológicos e interpessoais, que traz consigo uma carga enorme de preocupação, em especial quando acomete o segmento cefálico (sob a denominação de cefaleia), devido a sua significância orgânica, social e psicológica ${ }^{1}$.

$\mathrm{O}$ interesse no assunto e constante busca pelo seu alívio e melhor compreensão decorrem dos prejuízos acarretados por elas ${ }^{2,3}$. As cefaleias são consideradas um problema de saúde pública, uma vez que são desordens debilitantes e que, por vezes, impossibilitam ações rotineiras, o que causa um grande impacto pessoal e social ${ }^{4}$.

Trata-se do sintoma neurológico mais comum e de uma queixa médica muito frequente 5 . Apesar das variações regionais, cefaleias são um problema de distribuição mundial, envolvendo pessoas de todas as regiões, idades, 
raças e nível econômico ${ }^{6}$. Segundo a World Health Organization $(\mathrm{WHO})^{7}$, dores de cabeça serão sentidas, em algum momento, por quase todas as pessoas, sendo que metade dos adultos no mundo já experimentou pelo menos um dos três tipos mais comum de cefaleia. Acredita-se que cerca de $80 \%$ da população sofre com esta enfermidade a cada ano, sendo que somente em 10 a $20 \%$ dos casos um médico é consultado, o que faz com que a minoria das pessoas receba um diagnóstico apropriado, tornando a doença subestimada, tanto em reconhecimento quanto em tratamento, em todo o mundo ${ }^{1,6}$. Além disso, há o impasse do déficit de capacitação médica para diagnosticar a doença, observado na pesquisa de Galdino, Albuquerque e Medeiros $(2007)^{8}$, que encontraram que $82 \%$ dos médicos entrevistados (não-neurologistas que realizam atendimento primário a saúde) não tinham conhecimento da classificação e critérios diagnósticos das cefaleias primárias.

Atualmente se definem em mais de 100 tipos, apresentando etiologia variada, podendo decorrer de patologias estruturais, sistêmicas e/ou quadros disfuncionais, como, por exemplo, estados infecciosos, meningoencefalites, hemorragias intracranianas, hidrocefalia, tumores, lúpus eritematoso sistêmico, dentre outras ${ }^{5,9}$.

Apesar de sua prevalência e reconhecimento desde tempos antigos, até meados do século XX não havia clareza nem uniformidade quanto a sua classificação ${ }^{10}$. Foi somente em 1988 que a International Headache Society (IHS) ${ }^{11}$ propôs, através da publicação da "Classificação e Critérios Diagnósticos das Cefaleias, Nevralgias Cranianas e Dor Facial', um critério de classificação e diagnóstico das cefaleias, agrupando-as em dois grupos de acordo com sua etiologia: primárias e secundárias. As primárias têm sua origem no próprio segmento, não decorrendo de outras patologias, sendo válido ressaltar que, em geral, a dor costuma localizar-se no crânio e não na face. Já as secundárias são apenas sintomas consequentes de patologias estruturais. A título de exemplificação, enxaqueca com ou sem aura, cefaleia do tipo tensional (CTT) episódica ou crônica e cefaleia em salvas são alguns subtipos das cefaleias primárias; enquanto que cefaleia associada ao trauma, cefaleia associada com doenças metabólicas e cefaleia associada com substâncias ou sua retirada, subtipos das secundárias ${ }^{4,11,12}$.

\section{MATERIAL E MÉTODO}

Trata-se de uma revisão bibliográfica de 30 documentos, dentre eles artigos científicos, periódicos, livros e informações de organizações de referência mundial relacionados ao tema cefaleia, em especial a do tipo tensional. As buscas foram realizadas em bases de dados eletrônicos como: PubMed, Medline, Biblioteca Virtual em Saúde, Lilacs, SciELO, Google Acadêmico e arquivos da World Health Organization e International Headache Society. Tendo como critério de inclusão aqueles cujas informações foram tidas como de grande relevância, publicados em português, inglês ou espanhol; entre 1985 e 2016. Como critérios de exclusão, estipulou-se a retirada de artigos que apresentavam pouca ou nenhuma informação relevante ou relacionada ao tema, ou que não expunham subsídios adicionais aos outros dados já selecionados.

\section{REVISÃO DA LITERATURA}

A CTT é uma das doenças mais comuns da atualidade, apresentando uma ampla prevalência e um grande impacto social e econômico. Uma vez que traz, em maior ou menor grau, dependendo de seu subtipo, um comprometimento da qualidade de vida e da atividade dos acometidos, além de ser uma das morbidades mais onerosas da sociedade moderna ${ }^{13,14}$.

Apresenta-se ao longo da vida na população em geral sob a prevalência de 30 a $78 \%{ }^{14}$. Estudos de Goadsby e Boes ${ }^{15}$ obtiveram que a CTT representa $69 \%$ das dores de cabeça. Geralmente, inicia-se na adolescência, atingindo um pico por volta da terceira década de vida. Manifesta-se predominantemente em mulheres, em uma proporção de três mulheres a cada 2 homens, fato provavelmente decorrente de fatores de modulação hormonal ${ }^{4,6,7}$. Entre crianças e adolescentes representa a forma mais comum de cefaleia primaria $^{16}$.

A cefaleia do tipo tensional também é conhecida pelas denominações: cefaleia de tensão, cefaleia de contração muscular, cefaleia psicomiogênica, cefaleia do estresse, cefaleia comum, cefaleia essencial, cefaleia idiopática e cefaleia psicogênica ${ }^{14}$.

A IHS $^{14}$ através da terceira edição da International Classification of Headache Disorders (ICHD-III beta), classificou a CTT em alguns subgrupos, de acordo com a frequência e características das crises, conforme descrito na Tabela 1.

O nível de diagnóstico se dá pela quantidade de dígitos utilizados, sendo recomendável que médicos conheçam e utilizem de rotina a classificação, ao menos, de dois dígitos ${ }^{12}$.

Já na Classificação Internacional de Doenças (CID10), a CTT é codificada por G44.2 ${ }^{17}$. Seu quadro clínico é caracterizado por uma dor de localização frontal, parietal, temporal e/ou occipital, bilateral, com intensidade de leve a moderada, de caráter compressivo, podendo irradiar para a região cervical e não apresentando piora com esforço físico rotineiro. Apresenta duração variável de indivíduo para indivíduo ou em um mesmo indivíduo, relacionando-se, também, ao seu subtipo. Fatores associados como foto e fonofobia podem estar presentes, porém não há queixas de náuseas e vômitos ${ }^{3,4,7,10,14}$.

\section{DIAGNÓSTICO DIFERENCIAL}

Diferentes tipos de cefaleias primárias podem gerar confusão no momento do diagnóstico, de forma que sua distinção será possível através das características sintomatológicas de cada uma, baseando-se nos critérios da IHS $^{14,18}$. A CTT e a enxaqueca são dois tipos de cefaleias primarias, frequentemente correlacionadas, porém divergem com relação a impulsividade da dor, sendo vascular na enxaqueca e miofascial na cefaleia tensional. Além disso, apresentam clínica diferente, pois a enxaqueca manifesta-se com qualidade pulsátil, unilateral, com uma intensidade maior, associada a náuseas, vômitos e tontura, além de se agravar durante esforço físico. No entanto, muitas pessoas podem exibir um quadro de CTT e enxaqueca associados, condição que recebe a denominação de cefaleia mista. Esta se verifica em $62 \%$ dos enxaquecosos. A cefaleia mista tem início com a cefaleia, tipo tensional, que progride para um quadro de enxaqueca bem característico. Posteriormente, a cefaleia retoma ao tipo tensional, por mais algumas horas ou dias. Dessa forma, pode-se sugerir que a enxaqueca seja um fator predisponente da CTT, visto que episódios de cefaleia de tensão são mais frequentes e evidentes em indivíduos com enxaqueca ${ }^{4,5}$.

Há de se averiguar também se a dor não resulta do uso excessivo de analgésicos, cujo diagnóstico somente pode ser concluído retrospectivamente, ou seja, após a interrupção da droga e melhora do quadro do paciente ${ }^{18,19}$. 
Em sujeitos com episódios de cefaleias agudas de início ictal, recomenda-se uma avaliação imediata e uma investigação de patologias mais graves, em que a cefaleia caracterizaria um sintoma secundário. Para tanto, é imprescindível um exame neurológico completo, que, se anormal, será sucedido por um exame complementar tomografia computadorizada ou ressonância nuclear magnética. Em alguns casos, pode se tornar necessária uma punção lombar ou investigação dos aparelhos cardiovascular, renal, oftalmológico e/ou medular ${ }^{15}$.

Tabela 1. Classificação e subclassificação da CTT e critérios diagnósticos quanto à frequência e duração, segundo a ICHD-III ${ }^{14}$

\begin{tabular}{|c|c|c|}
\hline \multicolumn{3}{|c|}{ CEFALEIA TIPO TENSÃO } \\
\hline \multicolumn{2}{|c|}{ Subclassificação } & Frequência e Duração \\
\hline \multirow{2}{*}{$\begin{array}{l}\text { 2.1 Cefaleia tipo tensão } \\
\text { episódica pouco frequente }\end{array}$} & $\begin{array}{l}\text { 2.1.1 Cefaleia tipo tensão episódica pouco } \\
\text { frequente associada a dor pericraniana }\end{array}$ & \multirow[t]{2}{*}{$\begin{array}{l}\text { Mínimo de } 10 \text { episódios em um único dia } \\
\text { por mês - máximo de } 12 \text { dias por ano - cada } \\
\text { um durando de } 30 \text { minutos a } 7 \text { dias }\end{array}$} \\
\hline & $\begin{array}{l}\text { 2.1.2 Cefaleia tipo tensão episódica pouco } \\
\text { frequente não associada a dor pericraniana }\end{array}$ & \\
\hline \multirow[t]{2}{*}{$\begin{array}{l}\text { 2.2 Cefaleia tipo tensão } \\
\text { episódica frequente }\end{array}$} & $\begin{array}{l}\text { 2.2.1 Cefaleia tipo tensão episódica } \\
\text { frequente associada a dor pericraniana }\end{array}$ & \multirow{2}{*}{$\begin{array}{l}\text { Mínimo de } 10 \text { episódios que ocorrem em } 1 \text { a } \\
14 \text { dias, por mais de três meses - entre } 12 \text { e } \\
179 \text { dias/ano - cuja duração se dá entre } 30 \\
\text { minutos e uma semana }\end{array}$} \\
\hline & $\begin{array}{l}\text { 2.2.2 Cefaleia tipo tensão episódica } \\
\text { frequente não associada a dor pericraniana }\end{array}$ & \\
\hline \multirow{2}{*}{$\begin{array}{l}\text { 2.3 Cefaleia tipo tensão } \\
\text { crônica }\end{array}$} & $\begin{array}{l}\text { 2.3.1 Cefaleia tipo tensão crônica associada } \\
\text { a dor pericraniana }\end{array}$ & \multirow[t]{2}{*}{$\begin{array}{l}\text { Sentida } 15 \text { dias ou mais por mês ao longo de } \\
\text { mais de } 3 \text { meses - } 180 \text { dias ou mais, } \\
\text { anualmente - perdurando horas ou dias }\end{array}$} \\
\hline & $\begin{array}{l}\text { 2.3.2 Cefaleia tipo tensão crônica não } \\
\text { associada a dor pericraniana }\end{array}$ & \\
\hline \multirow{3}{*}{$\begin{array}{l}\text { 2.4 Cefaleia tipo tensão } \\
\text { provável }\end{array}$} & $\begin{array}{l}\text { 2.4.1 Cefaleia tipo tensão episódica pouco } \\
\text { frequente, provável }\end{array}$ & \multirow{3}{*}{$\begin{array}{l}\text { Abrange aquelas dores que não obedecem a } \\
\text { todos os critérios requeridos para os três } \\
\text { primeiros subtipos da CTT nem para outro } \\
\text { tipo de cefaleia }\end{array}$} \\
\hline & $\begin{array}{l}\text { 2.4.2 Cefaleia tipo tensão episódica } \\
\text { frequente, provável }\end{array}$ & \\
\hline & 2.4.3 Cefaleia tipo tensão crônica, provável & \\
\hline
\end{tabular}

\section{FISIOPATOLOGIA}

As estruturas cranianas capazes de gerar dor são: pele, couro cabeludo, artérias e veias de maior calibre (como artéria meníngea média e segmentos proximais das grandes artérias da pia-máter), seios durais, meninges, musculatura da cabeça e do pescoço, foice do cérebro, mucosa dos seios da face, articulações temporomandibulares e composições orais (dentes e gengiva) ${ }^{15,20}$

Por certo tempo, acreditava-se que a CTT era resultado de contrações prolongadas da musculatura pericraniana, que seriam respostas físicas a estímulos emocionais, psicológicos ou ambientais, razão pela qual também é conhecida como "cefaleia de contração muscular". Tal contração levaria a uma isquemia tecidual e, consequentemente, a um estímulo doloroso. Entretanto, atualmente é aceito que sua fisiopatologia, embora ainda não totalmente esclarecida, envolva mecanismos complexos, tanto periféricos quanto centrais de vias de produção da dor $^{2,4,5}$.

Mecanismos periféricos abrangem aumento da sensibilidade à palpação pericraniana e atividade eletromiográfica (EMG). O primeiro - presente tanto na CTT episódica, quanto na crônica - é entendido como uma dor induzida por pressão, em que a palpação dos tecidos miofasciais pericranianos, com consequente sensitização de fibras A-delta e C, responde com um aumento da sensibilidade.
Isquemia, estímulos mecânicos (como apertamento dentário) e mediadores químicos como serotonina, bradicinina e íons $\mathrm{K}+$ são alguns dos eventos desencadeadores de tal processo. Já estudos com EMG demonstraram que, na verdade, o que ocorreria não seria uma contração generalizada dos músculos, mas sim uma ativação prolongada e continua de algumas de suas unidades motoras, capazes de acionar os nociceptores periféricos, resultando em dor ${ }^{4}$.

Já os mecanismos centrais envolvem diversos fatores. O próprio elemento psicológico (estresse, depressão e ansiedade) apresenta íntima relação com a CTT, embora não se compreenda totalmente sua forma de ação ${ }^{4}$.

Outra possível explicação para o aparecimento deste tipo de cefaleia diz respeito ao limiar de detecção de dor induzida por pressão e ao limiar de tolerância a dor induzida por pressão, limites inferior e superior, respectivamente, de sensação dolorosa como resposta a um estimulo de pressão. Julga-se que, na CTT episódica não haja alteração no limiar de detecção da dor, enquanto que tem se verificado, na CTT crônica, uma diminuição do mesmo, juntamente com um limiar de tolerância a dor rebaixado, o que leva a alodínea e a hiperalgesia ${ }^{4}$.

Substâncias endógenas alteradas também são cogitadas como causadoras. Por exemplo, baixo nível de endorfina no líquor, que resultaria em alodínea prolongada ${ }^{2}$. 
Bem como níveis reduzidos de serotonina, um neurotransmissor participante do processo de modulação da dor, por apresentar uma ação antinociceptiva. Fato comprovado pela melhora da patologia através da administração de Amitriptilina (um antidepressivo tricíclico inibidor seletivo da receptação deste neurotransmissor) a indivíduos com CTT crônica. No entanto, ainda não se obteve confirmação quanto a relação entre CTT e os níveis plasmáticos e intraplaquetários de serotonina ${ }^{4,5}$.

Segundo Gómez ${ }^{18} 50 \%$ dos casos de CTT correlacionam-se a um aumento da pressão intracraniana do liquido cefalorraquidiano ou do sistema do sistema venoso do encéfalo.

A sensibilidade das regiões da face e do crânio é designada ao nervo trigêmeo ( $\mathrm{V}$ par craniano), que recebe a informação dolorosa, mas essa só é interpretada por estruturas como gânglios da base, sistema límbico e núcleos da rafe. Assim, a cronicidade da cefaleia pode decorrer de uma hipersensibilidade neuronal a diversos tipos de estímulos, facilitando a percepção da dor, de forma que incitações físicas, psicológicas ou mesmo contrações musculares tornam-se sensações álgicas ${ }^{2}$. Outra teoria seria a de que a dor decorreria de uma solicitação exagerada da membrana miofascial por constante contração muscular, resultando na liberação de toxinas, que, se acumuladas, afetariam terminações nervosas livres, gerando a dor ${ }^{1,5}$.

\section{FATORES DESENCADEANTES}

Fatores desencadeantes incluem, mas não se limitam a, mudanças dietéticas, déficit de hidratação, tensão elevada da musculatura da cabeça, pescoço e ombros, posições/postura inadequadas, ciclo menstrual, distúrbios do sono ou excesso dele, bruxismo, atividade mastigatória, consumo de determinados alimentos, bem como exposição a luz, odores fortes e barulhos ${ }^{4,21,22}$

Embora a relação causa-efeito não esteja totalmente esclarecida, fatores emocionais - tais como estresse, ansiedade e depressão - são o gatilho principal da CTT, em especial quando ocorrem de forma prolongada ${ }^{4,22}$.

Alterações climáticas com quedas bruscas de temperatura são cogitadas como possíveis causas da cefaleia tensional, devido ao aumento generalizado da tensão muscular resultante das baixas temperaturas, associado a uma menor ingestão hídrica, comum em períodos mais frios $4,21,22$

O consumo abusivo de determinadas substancias também pode desencadear a CTT: cafeína, por se tratar de uma substancia estimulante do Sistema Nervoso Central e desidratante; assim como analgésicos, tabaco e álcool ${ }^{4,22}$.

Fragoso et al. ${ }^{22}$ obtiveram em seu estudo que o ato de chorar teve uma ação potencial de gênese da cefaleia tensional em $55 \%$ dos casos analisados. Saueressig et al. ${ }^{23}$ concluíram que o uso exagerado de eletrônicos constitui um fator de risco para a CTT em adolescentes.

Pouco ainda é sabido sobre a hereditariedade da CTT, no entanto, Matta e Moreira Filho ${ }^{24}$ encontraram que $24 \%$ dos portadores de CTT entrevistados afirmaram ter parentes de primeiro grau com a doença; enquanto que Torelli et al. ${ }^{25}$ obtiveram histórico familiar positivo na maioria dos avaliados.

\section{PREVENÇÃO}

A cefaleia tensional é passível de prevenção através de atitudes que visam erradicar as possibilidades de desenvolvimento da doença, por meio, por exemplo, do controle do estresse ou desordens psíquicas, correção de condições inadequadas de alimentação, sono e postura, além do manejo de doenças dentárias e nos seios da face ${ }^{13}$.

\section{TRATAMENTO}

O tratamento da CTT se inicia com uma simples orientação do paciente acerca da natureza benigna da dor, tranquilizando-o e informando-o sobre a possibilidade e formas de tratamento. Nesta etapa, ainda, deve-se tentar descobrir os fatores desencadeantes das crises naquele individuo, que será orientado a evita-los ${ }^{4}$. Elabora-se, então, um plano terapêutico farmacológico e não farmacológico adequado e pautado em metas realistas ${ }^{9}$.

Ele pode ser sintomático, aquele de combate a fase álgica, ou profilático, cujo objetivo é reduzir as crises, tanto em número quanto em intensidade ${ }^{9}$. No sintomático recomenda-se evitar métodos farmacológicos, porém, se necessário, analgésicos comuns ou anti-inflamatórios não esteroides (AINEs) podem ser empregados. Já no profilático, geralmente se vale de medicamentos antidepressivos tricíclicos ${ }^{5,26}$.

Métodos não farmacológicos também podem ser utilizados e, por vezes, demonstraram-se mais efetivos. Envolvem um tratamento comportamental, em que o paciente é sujeito ativo de sua terapêutica, mudando seus hábitos quanto a sua alimentação, ingestão de água, consumo de substancias como cafeína e nicotina, postura, qualidade do sono e etc. Quando cabível, faz-se necessário um acompanhamento com uma equipe médica especializada para tratamento de ansiedade ou depressão ${ }^{4}$.

Se houver comprometimento muscular, indica-se tratamento fisioterápico. Embora esta terapia seja o meio não farmacológico mais recomendado e de maior aceitação cientifica para tratamento da CTT, não há protocolos fisioterapêuticos específicos definidos. Utilizam-se técnicas como: alongamentos, massoterapia, exercícios de relaxamento, reeducação postural, mobilizações passivas, crioterapia, treino de fortalecimento craniocervical, ultrassom e TENS ("Transcutaneous electrical nerve stimulation ou Neuroestimulação elétrica transcutânea"). Além disso, cabe ao fisioterapeuta orientar os doentes sobre como identificar, controlar, prevenir e lidar com os fatores rotineiros de estresse muscular e com a dor em $\mathrm{si}^{4,13}$.

Técnicas de relaxamento como massagens cervicais, aplicação de calor úmido local, banhos quentes de imersão, ioga e meditação transcendental, reduzem a tensão muscular e garantem uma melhora na qualidade de vida dos pacientes. Com a mesma finalidade e através dos mesmos mecanismos terapêuticos, também se tem disponível o bio-feedback ${ }^{4,5,15}$.

Atividade física, em especial com exercícios aeróbicos, libera endorfinas, analgésicos naturais, o que proporciona uma melhora na vascularização e oxigenação dos tecidos, bem como uma maior remoção de substâncias algógenas ${ }^{4}$.

Outras terapias se mostraram úteis no alivio dos sintomas desta patologia, em especial quando combinadas a outros métodos $^{13}$. Por exemplo, destaca-se a terapia cognitiva, auto-hipnose ou mesmo ferramentas da medicina alternativa, como do-in, acupressura e acupuntura, embora esta última divida opiniões e ainda não tenha comprovado sua real eficácia ${ }^{5,13,15}$.

Já para o tratamento farmacológico, as substâncias geralmente empregadas na etapa abortiva da dor são analgésicos, AINEs e relaxantes musculares; enquanto que antidepressivos tricíclicos são recomendados na CTT crônica $^{4,13}$. Os analgésicos mais utilizados são paracetamol e ácido acetilsalicílico ${ }^{15}$. O Ácido Mefenâmico também pode 
ser utilizado, em especial nos casos em que há correlação da dor com o ciclo menstrual. Os AINEs mais recomendados são o Ibuporfeno e o Naproxeno, sempre se respeitando as contraindicações destas drogas, como em casos de úlceras pépticas e gastrites erosivas ${ }^{4,5}$. Se estas medicações forem necessárias por um período prolongado, recomenda-se substituir o tratamento por um profilático, com antidepressivos tricíclicos ${ }^{26}$. Deve-se salientar o perigo do abuso de analgésicos, especialmente aqueles que combinam aspirina, dipirona, acetaminofeno e cafeína opióides, ergotamina ou triptanos - pois pode ocasionar uma cefaleia rebote ou cefaleia por abuso de medicação. Nestes casos, deve-se suspender repentinamente a droga e iniciar medicação profilática específica ${ }^{4,19}$.

Nos casos crônicos, emprega-se os antidepressivos tricíclicos, sendo a droga de primeira escolha é a Amitriptilina, associada ou não a relaxantes musculares, como Tizanidina, Carisoprodol e Ciclobenzaprina ${ }^{4,13,19}$. Nortriptilina ou Imipramina também podem ser utilizadas ${ }^{26}$.

Há controvérsias com relação aos efeitos da toxina botulínica na terapêutica da cefaleia, tipo tensional. Varjão et al. ${ }^{4}$ afirma que esta é sim uma alternativa vantajosa para casos refratários, em que os outros mecanismos terapêuticos falharam, e sugere que seus benefícios provenham de uma interrupção transitória entre a nocicepção periférica e o processamento central da dor. Outros dois estudos com toxina botulínica tipo A observaram uma redução na frequência das dores de cabeça nos casos em que esta foi utilizada $^{27,28}$. Entretanto, alguns autores acreditam que seu emprego não trata efetivamente a $\mathrm{CTT}^{15,29,30}$. Borges et al. ${ }^{30}$ obtiveram em seu estudo que a toxina botulínica apenas controla temporariamente a cefaleia, mas que traz consigo também efeitos colaterais como hiperatividade e dor muscular.

\section{CONCLUSÃO}

Conclui-se que a CTT é uma patologia de alta incidência mundial e traz consigo prejuízos sociais e econômicos. Na grande maioria dos casos seu diagnóstico e tratamento são realizados por médicos generalistas, que, por vezes, apresentam conhecimento deficitário sobre a ICHDIII beta, o que dificulta a formulação de um plano terapêutico correto e implica em uma redução acentuada da qualidade de vida dos pacientes. Apresenta fisiopatologia complexa e multicausal, sendo que o melhor tratamento envolve ações não farmacológicas, porém medicamentos podem auxiliar na melhora e na profilaxia dos quadros. É passível de prevenção se adotadas medidas simples que evadiram seus fatores desencadeantes.

\section{REFERÊNCIAS}

1. Petersen CS, Nunes MLT. Cefaleia tensional crônica e psicopatologia. Psic. 2002; 3(2):30-43.

2. Flores AMN, Costa Junior AL. Modelo biopsicossocial e formulação comportamental: compreendendo a cefaleia do tipo tensional. Psicol estud. 2008; 13(1):14351.

3. Flores AMN, Costa Junior ALC. O manejo psicológico da dor de cabeça tensional. Psicol ciênc prof. 2004; 24(3):24-33.

4. Varjão FM, Jorge JH, Nepelenbroek KH, Alencar Júnior FGP. Cefaleia, tipo tensional. Saúde e Pesquisa. 2008; 1(2):185-91.

5. Sanvito WL, Monzillo PH. cefaleias primárias: aspectos clínicos e terapêuticos. Medicina. 1997; 30:437-48.
6. World Health Organization (WHO). Fact sheet: headache disorders. Genebra, 2016. disponível em: http://www.who.int/mediacentre/factsheets/fs277/en/. acesso em: 5 jul de 2016.

7. World Health Organization (WHO). ATLAS of Headache Disorders and Resources in the World 2011. Genebra, 2011. Disponível em: http://www.who.int/ entity/ mental_health/management/ who_atlas_headache_disorders.pdf?ua=1. Acesso em: 7 jul 2016.

8. Galdino GS, Albuquerque TIP, Medeiros JLA. Cefaleias primárias: Abordagem diagnóstica por médicos nãoneurologistas. Arq Neuro-Psiquiatr. 2007; 65(3-A):681-4.

9. Gherpelli JLD. Tratamento das cefaleias. J Pediatr. 2002;78(supl.1):S3-S8.

10. Perla AS. Cefaleias: diagnóstico diferencial. In: Chaves MLF, Finkelsztejn A, Stefani MA (Eds.). Rotinas em neurologia e neurocirurgia. $1^{a}$ Ed. Porto Alegre: Artmed; 2008. p. 128-143.

11. Classification and diagnostic criteria for headache disorders, cranial neuralgias and facial pain. Headache Classification Committee of the International Headache Society. Cephalalgia. 1988; 8(Suppl 7):1-96.

12. Speciali JG. Classificação das cefaleias. Medicina. 1997;30: 421-7.

13. Santos CMT, Pereira CU, Lima PTMR, Santos EAS, Monteiro JTS. Cefaleia tensional. RBM. 2008; 65(5):112-7.

14. Headache Classification Committee of the International Headache Society (IHS). The International Classification of Headache Disorders, 3rd edition (beta version). Cephalalgia. 2013; 33(9): 629-808.

15. Goadsby PJ, Raskin NH. Cefaleia. In: Hauser SL, Josephson SA. Neurologia clínica de Harrison. 3. Ed (Edição Digital). Porto Alegre: AMGH; 2015. p. 41-56.

16. Siqueira LFM. Cefaleias na infância e adolescência. Pediatr Mod. 2011; 47(1):5-12.

17. World Health Organization. International Statistical Classification of Diseases and Related Health Problems. 10th revision, Fifth edition, 2016. Disponível em: http://apps.who.int/classifications/icd10/browse/2016/en

18. Gómez MV. Cefaleia tipo tensional: diagnóstico, fisiopatologia y tratamento. Acta Neurol Colomb. 2008; 24(3) Supl. (3:1).

19. Stancioli FG, Vasconcelos LPB, Leal JC, Silva Júnior AA, Gomez RS, Teixeira AL. Cefaleia Crônica Diária. RBM. 2007; 64(1/2):5-10.

20. Aquino JHW, Fortes FM. Cefaleias na adolescência. Adolesc Saúde. 2009: 6(3):35-40.

21. World Headache Alliance (WHA). Tension Type Headache, 2016. Disponível em: http://www.w-h-a.org/. Acesso em 5 jul 2016

22. Fragoso YD, Carvalho R, Ferrero F, Lourenço DM, Paulino ER. Crying as a precipitating factor for migraine and tension-type headache. Sao Paulo Med. J. 2003: 121(1): 31-3.

23. Saueressig IB, Xavier MKA, Oliveira VMA, Pitangui ACR, Araújo RC. Primary headaches among adolescents and their association with excessive computer use. Rev Dor. 2015; 16(4):244-8.

24. Matta APC, Moreira Filho, PF. Cefaléia do Tipo Tensional Episódica: Avaliação clínica de 50 pacientes. Arq Neuro-Psiquiatr. 2006; 64(1):95-9.

25. Torelli P, Cologno D, Manzoni GC. Weekend headache: a retrospective study in migraine without aura and 
episodic tension type headache. Headache 1999; 39(1):11-20.

26. Figuerola ML. Cefaleas primarias. In: Micheli F, Pardal MF. Neurología. 2. ed. Buenos Aires: Médica Panamericana; 2010. Cap. 6, p. 131-142.

27. Mathew NT, Frishberg BM, Gawel M, Dimitrova R, Gibson J, Turkel C, BOTOX CDH. Study Group. Botulinum toxin type A (BOTOX) for the prophylactic treatment of chronic daily headache: a randomized, double-blind, placebo-controlled trial. Headache. 2005; 45(4):293-307.

28. Silberstein SD, Stark SR, Lucas SM, Christie SN, Degryse RE, Turkel CC, BoNTA-039 Study Group. Botulinum toxin type A for the prophylactic treatment of chronic daily headache: a randomized, double-blind, placebo-controlled trial. Mayo Clin Proc. 2005; 80(9):1126-37.

29. Schulte-Mattler WJ, Martinez-Castrillo JC. Botulinum toxin therapy of migraine and tension-type headache: comparing different botulinum toxin preparations. Eur $\mathbf{J}$ Neurol. 2006; 13(Suppl 1):51-4.

30. Borges RN, Melo M, Barcelos BA, Carvalho Júnior H, Santos ARBR, Honorato ISS. Efeito da toxina botulinica na terapeutica da cefaleia tipo tensional. Rev Odontol Bras Central 2013;21(61): 85-9.

\section{CONFLITO DE INTERESSES}

Os autores declaram não haver conflitos de interesse.

\section{AUTOR PARA CORRESPONDÊNCIA}

Marlene Cabral Coimbra da Cruz

mcoimbracruz@gmail.com

Submetido em 26/10/2016 Aceito em 05/12/2016 